\title{
USING BARS AS SIGNPOSTS OF GALAXY EVOLUTION AT HIGH AND LOW REDSHIFTS
}

\author{
Kartik Sheth ${ }^{1}$, Karin Menendez-Delmestre ${ }^{1}$, Nick Scoville ${ }^{1}$, Tom Jarett ${ }^{1}$, Linda \\ Strubbe ${ }^{1,2}$,Michael W. Regan ${ }^{3}$, Eva Schinnerer ${ }^{4}$, and David Block ${ }^{5}$ \\ ${ }^{1}$ California Institute of Technology, Pasadena, CA, ${ }^{2}$ University of California - Berkeley, ${ }^{3}$ Space \\ Telescope Science Institute, ${ }^{4}$ National Radio Astronomy Observatory, ${ }^{5}$ University of the Wit- \\ watersrand
}

\begin{abstract}
An analysis of the NICMOS Deep Field shows that there is no evidence of a decline in the bar fraction beyond $\mathrm{z} \sim 0.7$, as previously claimed; both bandshifting and spatial resolution must be taken into account when evaluating the evolution of the bar fraction. Two main caveats of this study were a lack of a proper comparison sample at low redshifts and a larger number of galaxies at high redshifts. We address these caveats using two new studies. For a proper local sample, we have analyzed 134 spirals in the near-infrared using 2MASS (main results presented by Menendez-Delmestre in this volume) which serves as an ideal anchor for the low-redshift Universe. In addition to measuring the mean bar properties, we find that bar size is correlated with galaxy size and brightness, but the bar ellipticity is not correlated with these galaxy properties. The bar length is not correlated with the bar ellipticity. For larger high redshift samples we analyze the bar fraction from the 2-square degree COSMOS ACS survey. We find that the bar fraction at $\mathrm{z} \sim 0.7$ is $\sim 50 \%$, consistent with our earlier finding of no decline in bar fraction at high redshifts.
\end{abstract}

\section{Bars: Signposts of Galaxy Evolution}

Bars are ubiquitous in local disk galaxies (e.g., RC3, Eskridge et al. 2000, Menendez-Delmestre et al. 2004). They play an important role in evolving galaxies by transporting vast amounts of gas to the center (Sakamoto et al. 1997; Sheth et al. 2004), igniting circumnuclear starburst activity (Ho et al. 1997 and references therein), reducing the chemical abundance gradient (Martin \& Roy 1997), and perhaps feeding black holes (Shlosman et al. 1988, see Knapen and Laurikainen in this volume). Bars also form bulges, and perhaps evolve galaxies along the Hubble sequence (e.g., Norman, Sellwood \& Hasan 1996; see also Kormendy, Bournaud, and Combes in these proceedings). At high redshifts bars were expected to be fairly common because of dynamically 
colder disks and increased merging activity. So it was a surprise when the first studies of high redshift galaxy morphology found an apparent paucity of barred spirals beyond z 0.5-0.7 (van den Bergh et al. 1996; Abraham et al. 1999; van den Bergh et al. 2000). If true these results set strict constraints on cosmological simulations of galaxy formation by requiring that disks were not sufficiently massive, or were dynamically too hot until only $\sim 6$ Gyr ago. These constraints would be in stark contrast to conclusions from studies of the cosmic star formation history (e.g., Steidel et al. 1999 and references therein) and thinness of local disks (e.g., Toth \& Ostriker 1992) which argue that massive disks were already in place by $\mathrm{z} \sim 1$.

Bunker (1999) used a near-infrared 1.6 $\mu \mathrm{m}$ NICMOS GTO image to show that in at least one galaxy, a bar was missed by the early studies because they observed the high redshift galaxies in the rest-frame blue / ultraviolet light where bars are difficult to identify. The increasingly better visibility of bars at longer wavelengths is a well-known effect (see for example Figure 1, and Menendez-Delmestre et al and references therein in these proceedings). We decided to investigate whether the apparent decline in the bar fraction at high redshifts could be due to such a selection effect? This question was the focal point of Sheth et al. (2003) and whose results we briefly summarize here.

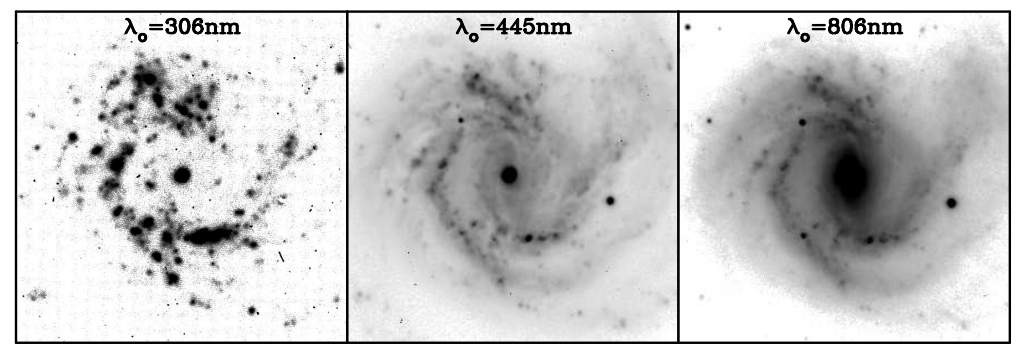

Figure 1. Left panel: UV appearance of NGC 4303, simulated using a continuum-subtracted $\mathrm{H} \alpha$ image. Note how the bar is completely invisible. Middle panel: Blue-band image. The bar is faint and difficult to identify. Right panel: I-band image. Only now does the bar become visible.

\section{The Bar Fraction in the NICMOS Deep Field}

We analyzed the bar fraction and bar properties in the WFPC2 (V, and Iband) and NICMOS (H-band data) for the northern Hubble Deep Field (Williams et al. 1996; Dickinson et al. 2000). The NICMOS data are ideal for studying galaxies in the redshift range $0.7, \mathrm{z}<1$ because these data observe the galaxies in rest-frame $\mathrm{V}$ through I-band light. 


\section{The Bar Identification Technique}

In contrast to previous studies which identified bars by eye, by ellipticity breaks, or a change in isophote position angles between an inner and outermost isophote (e.g., van den Bergh 1996; Abraham et al. 1999;), we applied a more stringent two signature criteria to identify bars. We demanded that a) the ellipticity increase monotonically and then drop with a sharp change of at least $\Delta \epsilon>0.1$, and b) the position angle remain constant over the bar region and change by $\Delta \mathrm{PA}>10^{\circ}$ after the bar region. We require that the bar be symmetric by fixing the center for the galaxy fitting algorithm. Our algorithm misses bars if the galaxy is highly inclined, if the bar position angle is the same as the galactic disk, if the underlying galactic disk is too faint to be adequately imaged, or if the data have inadequate resolution to resolve bars. Our method, though perhaps overly strict, has the advantage of ensuring a firm lower limit to the bar fraction.

As discussed in Sheth et al. (2003), at $\mathrm{z}<0.7$, we identify five barred spirals, and two candidate barred spirals, consistent with the prior analysis of the HDFN by Abraham et al. (1999). At $z>0.7$ we identify four barred spirals and five candidate barred spirals, including two possible candidates at $\mathrm{z}=1.66$ and $z=2.37$. The four barred spirals are shown in Figure 2. For comparison, in the previous WFPC2 HDFN studies, van den Bergh et al. (1996) found no barred spirals, and Abraham et al. (1999) found two barred galaxies beyond $\mathrm{z} \sim 0.5$. If we use the Abraham et al. (1999) magnitude cutoff of $\mathrm{I}(\mathrm{AB})=23.7$, the total number of disk-like galaxies drops to 31 ; amongst these we identify three barred spirals. Though we detect a few more bars, the total number of barred spirals is still small. Does this reflect a true decline in the bar fraction at $\mathrm{z}>0.7$ ? We argue that when the spatial resolution of the observations are considered in the context of bar visibility, there is no evidence of a decline in bars at $\mathrm{z}>0.7$.

\section{Spatial Resolution \& the Visibility of Bars}

In Figure 3, we show the apparent angular size of various galactic structures as a function of redshift. Overlaid are detection limits for various telescopes adopting a five PSF detection threshold; five PSFs is an appropriate choice (Menendez-Delmestre et al. 2004, also in this volume). The figure shows that at $0.8 \mu \mathrm{m}$ WFPC 2 data is only marginally capable of detecting a $5 \mathrm{kpc}$ structure beyond $\mathrm{z} \sim 0.7$. The NICMOS data have even coarser resolution (longer $\lambda$ and larger pixels), and even though these data are not affected by bandshifting until $\mathrm{z}>2-3$, they only detect structures with sizes $\gtrsim 10 \mathrm{kpc}$. The average size of the four bars identified at $\mathrm{z}>0.7$ is $12 \mathrm{kpc}$.

The most important point of note from Figure 3 is that a measurement of the bar fraction must take into account the size of bars and the available spatial res- 

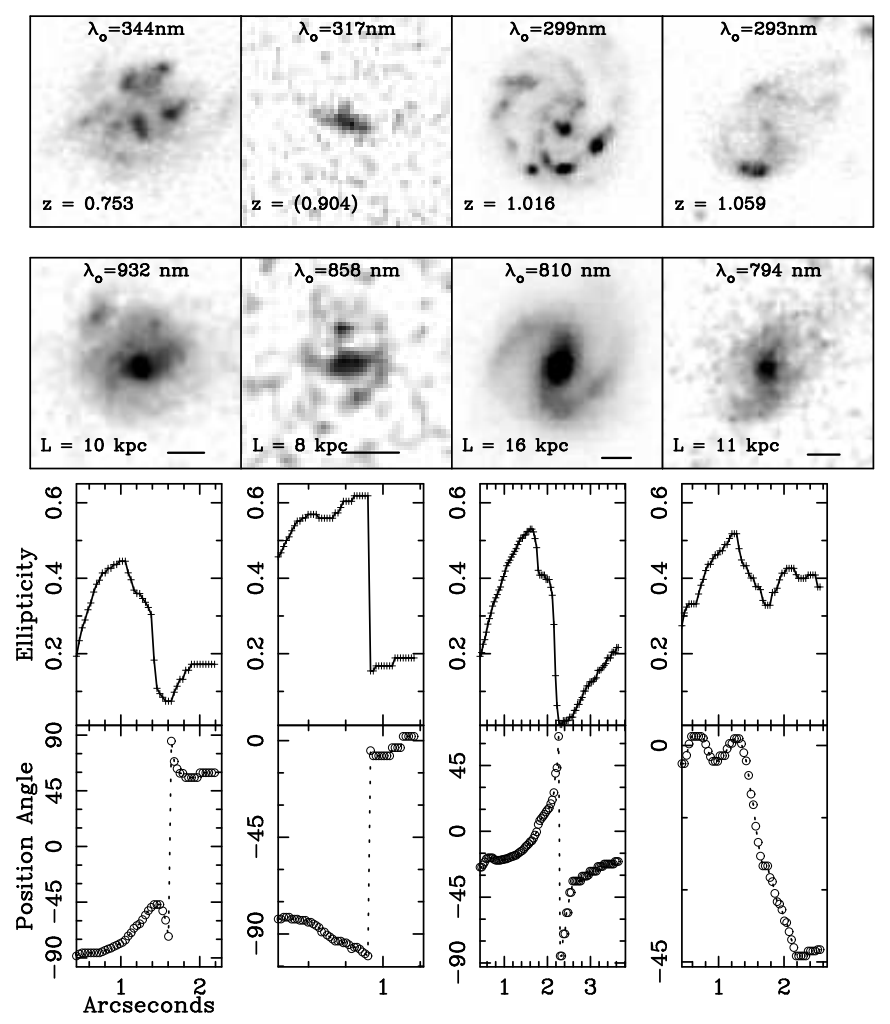

Figure 2. Barred spirals at $\mathrm{z}>0.7$, arranged by redshift. The top row shows the optical (F606W, V-band) WFPC2 images and the second row shows the near-infrared (1.6 $\mu \mathrm{m}, \mathrm{H}$-band) NICMOS images. 0.5" scale is shown with a horizontal segment in the lower right of each panel. The rest-frame wavelength for each galaxy is listed inside the top of each panel.

olution of the data. Although we only detect three or four bars in the NICMOS data one must note that these data are biased towards detecting only the largest bars. Therefore, when we compare the bar fraction at different redshifts, we must compare the fraction for bars of equal sizes. For a representative sample of local galaxies (SONG, Regan et al. 2001, Sheth et al. 2004), we find that bars with sizes $>12 \mathrm{kpc}$ are rare; only one out of 44 galaxies in SONG has a bar larger than $12 \mathrm{kpc}$. Thus the fraction of bars we detected in the NICMOS Deep Field (4/95 galaxies for the entire sample, or 3/31 galaxies using an I-magnitude cutoff) is similar, and perhaps even, larger than the bar fraction seen in SONG. Thus we concluded that there was no evidence for a decline in the bar fraction at $\mathrm{z}>0.7$, as previously claimed.

Our results are hampered by small number statistics (we discovered only a handful of bars at $\mathrm{z}>0.7$ and our local comparison sample contained forty four galaxies), and difficulty in defining comparable samples at high and low 


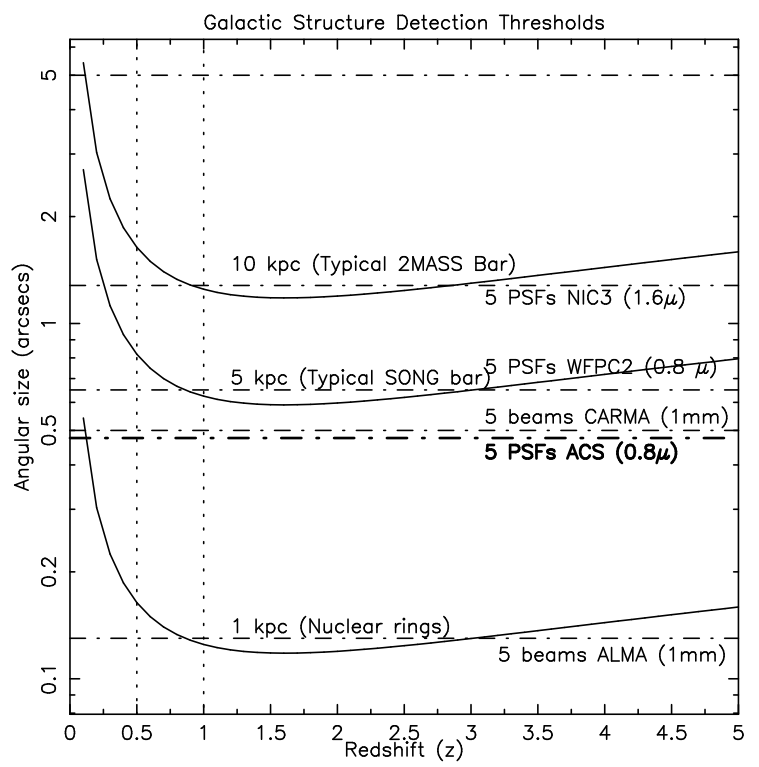

Figure 3. The detection threshold of various galactic structures as a function of redshift for different telescopes and instruments is shown. The horizontal dotted-dashed lines are an arbitrary 5 PSF or 5 beam limit. Note that at $0.8 \mu \mathrm{m}$, even the WFPC2 data is only marginally capable of detecting a typical $5 \mathrm{kpc}$ bar at $\mathrm{z}>0.7$; ACS z-band is only slightly better. The NICMOS data can only detect the large bars at $\mathrm{z}>0.5$. Also shown are capabilities of two new millimeter arrays, CARMA and ALMA which will be ideal for probing the gas kinematics in high redshift systems.

redshifts (see Sheth et al. 2003). From the existing analysis, it is difficult to conclusively state how the bar fraction varies as a function of redshift, and how the bar properties vary. In the next two sections we outline new results from two separate on-going studies aimed at overcoming these two main caveats of our NICMOS study.

\section{Defining a Proper Local Sample: The 2MASS Local Galaxy Atlas}

How well do we know the fraction of nearby bars? What are their properties (size, strength)? How do these properties depend on the host galaxy properties? Answers to these questions are of fundamental importance before any comparison is made to the high redshift Universe.

Since bars are best studied in the infrared, we decided to address all of these questions using the 2MASS Large Galaxy Sample. Results of this study were presented in a poster at this conference by Menendez-Delmestre. We chose all spirals of type $\mathrm{Sa}-\mathrm{Sd}$, with $i<65^{0}$ for a sample of 134 galaxies in $\mathrm{J}+\mathrm{H}+\mathrm{K}$. We 
ran the same ellipse fitting algorithm on these galaxies as we did previously on the high redshift sample and identified bars using the two signature criteria described above.

As shown in Figure 2 of Menendez-Delmestre et al. (this volume), the fraction of barred spirals in the 2MASS sample is $58 \%$ (see Figure 1 in MenendezDelmestre et al.). Another $21 \%$ of the galaxies are identified as candidate bars where usually there is an ellipticity signature but no corresponding position angle change. We examined each of the candidate bars by eye and found that about two-thirds of the candidates were in fact barred. So the total fraction of barred spirals in the infrared $\sim 72 \%$, similar to the fraction found by Eskridge et al. (2000). The bar fraction changes slightly with the T-type with the highest fraction $(80 \%)$ in $\mathrm{T}=3$ but there is no significant trend in the fraction with the Hubble type. Unfortunately the 2MASS sample has low signal to noise and we were unable to quantify the bar fraction in galaxies of type later than Sd. Note that the overall bar fraction in RC3 is lower, but not significantly different than the 2MASS fraction. This suggests that overall the change in the bar fraction between B-band (RC3) and the near-infrared is not large but also note that the effect may become severe at wavelengths shorter than the B-band, as shown by Figure 1.

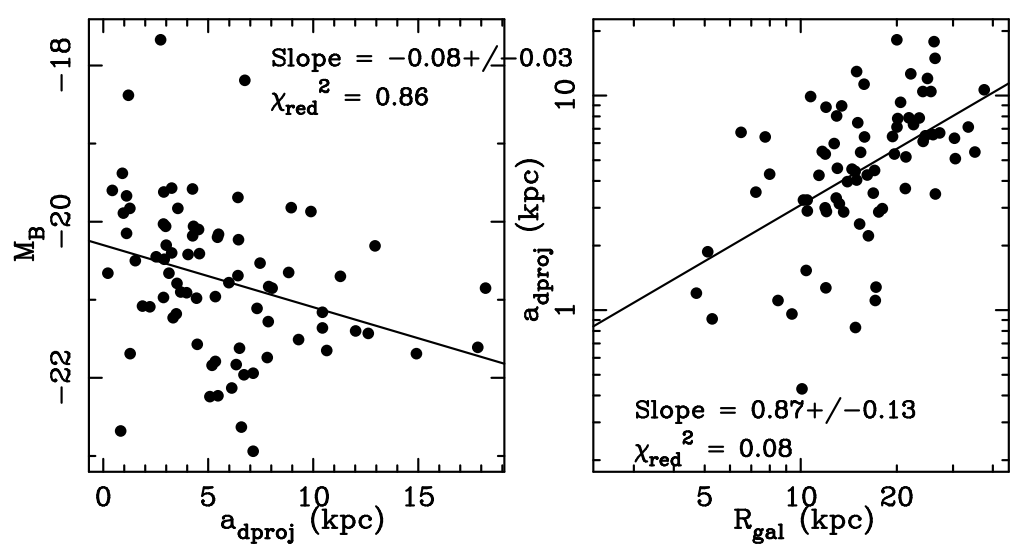

Figure 4. Left Panel: Absolute B-magnitude vs. deprojected bar semi-major axis. Right Panel: Deprojected bar semi-major axis vs. galaxy size. There are weak trends of larger bars in brighter and larger galaxies.

For the 78 barred spirals in 2MASS, we derive a median deprojected bar semi-major axis of $5.1 \mathrm{kpc}$. This result is different from the SONG sample we considered in Sheth et al. (2003). The reason for the difference is that 2MASS surveys a larger volume but is shallower. As a result we observe larger and brighter galaxies which tend to have longer bars (see below). We measure a mean ellipticity of 0.45 but the most notable result from the distribution of 
ellipticities is the scarcity of thin bars $(\epsilon>0.7)$. Previously similar results have been interpreted as evidence for a second or later generation of bars (e.g., Block et al. 2002, see also Bournaud, Combes in this volume) because in numerical simulations the first generation of bars is expected to be strong (thin) whereas subsequent generations are expected to be weaker (fatter). However note that the measurement of ellipticity can be affected by bulge light. Ellipse fitting does not automatically account for bulge light and as a consequence gives a higher ellipticity than the actual value. When we compare the bar ellipticity and bar length we find no correlation indicating that larger bars are preferentially fatter or thinner. We find that bars come in all shapes and sizes.

How do the bar properties depend on the host galaxy? From the 2MASS data, we find a trend of larger bars in larger and brighter galaxies (Figures 4). This is expected given that the bar instability is correlated with the mass of the disk.

Correlations of the bar strength (we use ellipticity as a proxy for strength) and the host galaxy parameters are very weak (Figures 5). Unlike the bar length, there is no correlation between the bar ellipticity and galaxy size or brightness.
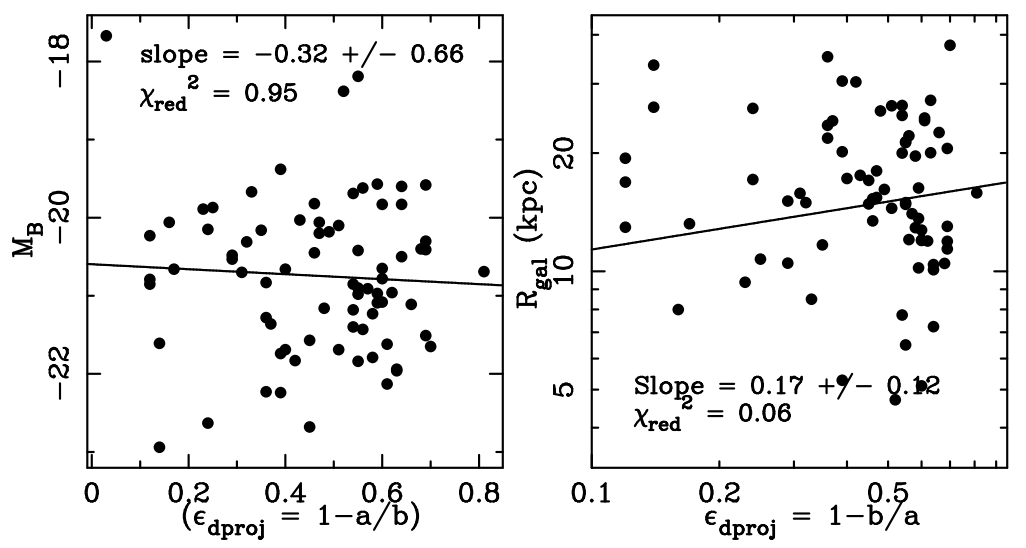

Figure 5. Left Panel: Absolute B-magnitude vs. deprojected bar ellipticity. Right Panel: Size of galaxy vs. deprojected bar ellipticity. There is no correlation between the bar ellipticity and the host galaxy size or brightness.

Note that these correlations are not immune to biases in the analysis. For instance the ellipse fitting technique always underestimates the bar ellipticity (Sheth et al. 2000, 2004) because the bulge light affects the galaxy isophotes to make the fitted ellipses fatter/thicker than the real underlying bar. Hence the lack of "strong" bars in early-Hubble type galaxies is not necessarily a real effect. Another bias is related to resolution. Small bars are difficult to identify especially in galaxies with bright bulges and nuclei. So any trend of larger 
bars in earlier type galaxies should also be viewed with caution. Nevertheless the trends of larger bars in larger galaxies and brighter galaxies are relatively robust.

\section{COSMOS: Overcoming Small Number Statistics}

The 2MASS analysis by Menendez-Delmestre et al. (2004) offers an excellent analysis of the local sample of bars and their host galaxies. We can now extend these results to higher redshifts to understand how the bar fraction and bars evolved over time.
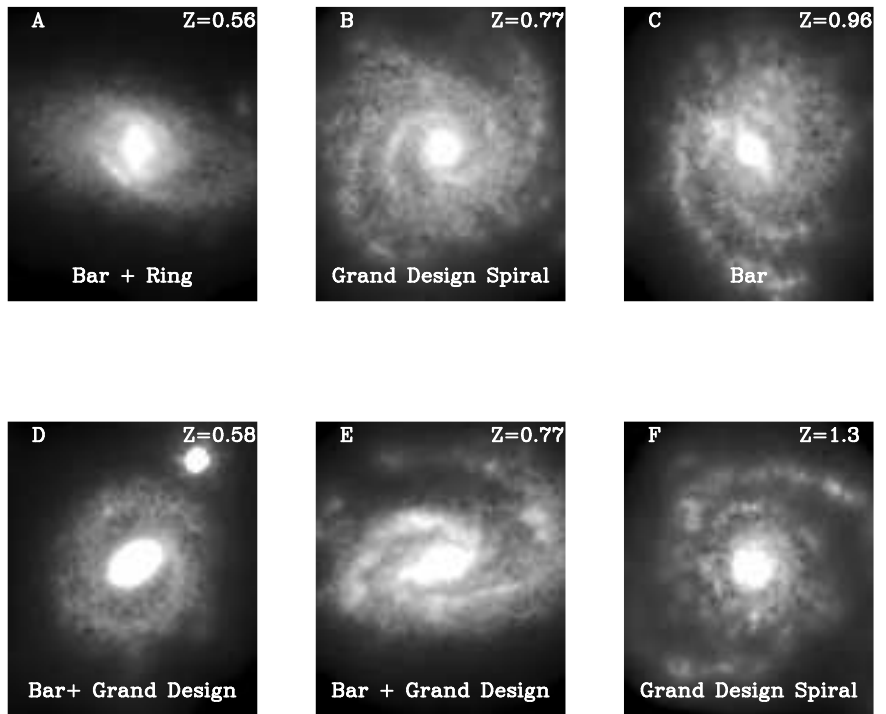

Figure 6. Typical barred and spiral galaxies from COSMOS data. Galaxy labeled A is virtually a twin of NGC 4303 shown in Figure 1. It shows a strong bar and a ring encircling the bar. The redshifts are photometrically derived from ground-based BVRIz Subaru data and are reliable to z 0.8 . So galaxies $\mathrm{C}$ and $\mathrm{F}$ may in fact be at lower redshifts than indicated here.

Until recently, the Hubble Deep Fields were the standard windows into the high redshift Universe. These data already indicated that defining a comparative sample at high redshifts is difficult. For example the fraction of irregular objects increases to nearly $30 \%$ to z 1 (e.g., Griffiths et al. 1994, Abraham et al. 1996). Lilly et al. (1996) concluded that disks evolve passively in luminosity whereas Shude et al. (1998) argue in favor of significant evolution. Certainly some of these discrepancies are a result of cosmic variance and scarcity of high quality data. These gaps, however, are now being filled with surveys like GOODS, UDF, DEEP2, and GEMS.

The most ambitious and revolutionary new survey is COSMOS (http://www.astro.caltech.edu/cosmos) which is observing a contiguous 2-square degree equatorial field using the Ad- 
vanced Camera for Survey on the Hubble Space Telescope, and multi-wavelength data from X-rays to radio. The size of the field is important because it overcomes cosmic variance and provides a truly representative view of the high redshift Universe. COSMOS is an ideal treasury program with a vast range of applications. In particular, for the study of galaxy morphology COSMOS is a gold mine (or more appropriately for this conference, a diamond mine) of opportunity. It will provide spectroscopic redshifts for over 100,000 galaxies with 10,000 galaxies in each of five bins of from $\mathrm{z}=0.5$ to $\mathrm{z}=2$. The spatial resolution of ACS data is exquisite. As shown in Figure 3, one can easily identify structures with sizes as small as $3 \mathrm{kpc}$ in diameter. If Figure 6 we show some typical examples of disk galaxies at three photometrically determined redshifts.

\section{The Bar Fraction in COSMOS}
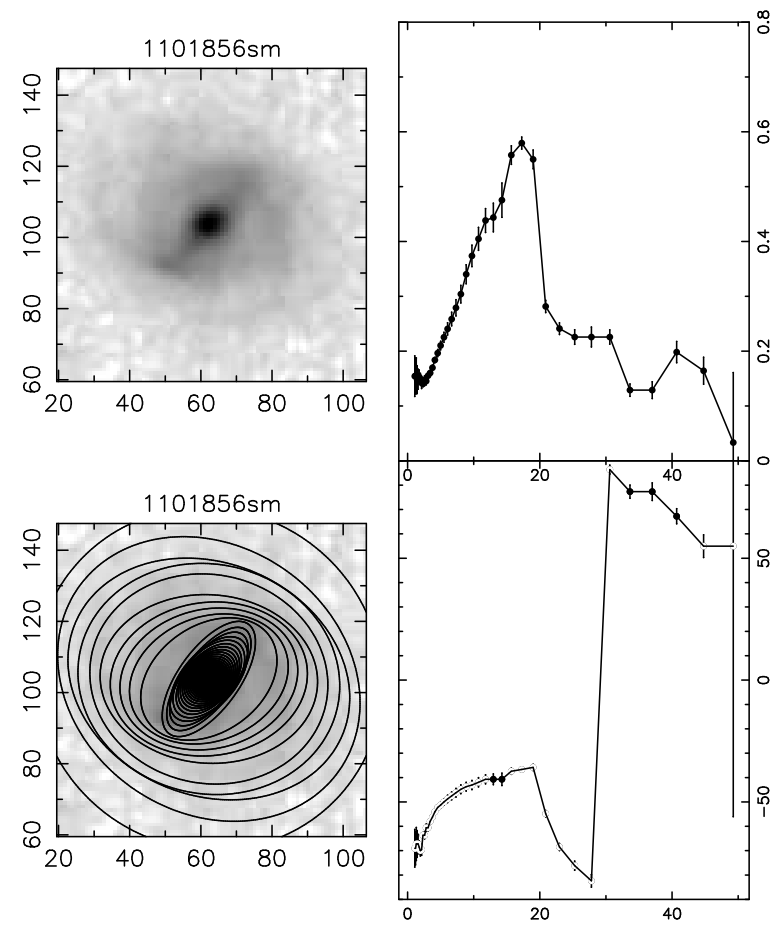

Figure 7. Example of ellipse fitting for a barred spiral in COSMOS.

In order to image such a large field with adequate sensitivity (COSMOS is within half a magnitude of the GOODS data) we chose to use the broader Iband filter over the z-band filter for COSMOS. Hence the analysis of the bar fraction can be done with full confidence to $\mathrm{z} \sim 0.7-0.8$ and perhaps to even 
higher redshifts. To date approximately 200 fields out of 600 have been observed. For these fields we first identified a sample of spiral galaxies from a photometric catalog derived from BVRIz data from observations on the Subaru teelscope for the entire COSMOS field. We only chose those galaxies that had photometric redshifts derived with the highest confidence $(>95 \%)$. We ran the same ellipse fitting algorithm on these galaxies. An example of such a fit is shown in Figure 7 . We have done a preliminary analysis for the redshift bin $0.6<\mathrm{z}<0.8$ for $\sim 700$ galaxies. We find that the fraction of barred spirals is $\sim 50 \%$, consistent with the local fraction. Although further analysis is necessary with better redshifts and the complete sample, the results already indicate that the bar fraction is not declining significantly, at least out to $\mathrm{z} \sim 0.8$.

\section{References}

Abraham, R. G., Tanvir, N. R., Santiag o, B. X., Ellis, R. S., Glazebrook, K., van den Bergh, S. 1996, MNRAS, 279, L47

Abraham, R. G., Merrifield, M. R., Ell is, R. S., Tanvir, N. R., \& Brinchmann, J. 1999, MNRAS, 308, 569

Block, D.L., Bournard, F., Combes, F., Puerari, I., \& Buta, R. 2002, A\&A, 394, 35

Bunker, A,J. 1999, in "Photometric Redshifts and the Detection of High Redshift t Galaxies", ASP Conference Series, Vol. 191, Eds. Weymann, R., Storrie-Lombard i, L., Sawicki, M., \& Brunner, R.

Dickinson, et al. 2000, ApJ, 531, 624

Eskridge, P., et al. 2000, AJ, 119, 536

Griffiths, R. E. et. al. 1994, ApJL, 435, 19

Ho, L. C., Filippenko, A. V., \& Sargent, W. L. W. 1997, ApJ, 487, 591

Lilly, S., Lefevre, O., Hammer, F., Cramp ton, D., Schade, D. J., Hudon, J. D., Tresse, L. 1996, IAUS, 171, 209

Martin, P., \& Roy, J. 1994, ApJ, 424, 599

Norman, C. A., Sellwood, J. A., \& Hasan, H. 1996, ApJ, 462, 114

Regan, M. W., Helfer, T. T., Thornley, M. D., Sheth, K., Wong, T., Vogel, S. N., Blitz, L., \& Bock, D. C.-J. 2001, ApJ, 561, 218

Sakamoto, K., Okumura, S. K., Ishizuki, S., Scoville, N. Z. 1999, ApJ, 525, 691

Sheth, K., Regan, M.W., Vogel, S.N., \& Teuben, P.J. 2000, ApJ, 532, 221

Sheth, K., Regan, M.W., Scoville, N.Z., \& Strubbe, L.E. 2003, ApJL, 592, 13

Sheth, K., Vogel, S. N., Regan, M. W., Teuben, P.J., Harris, A. I., Thornley, M. D., \& Helfer, T.T. 2004, ApJ, submitted

Shude, M., Mao, H. J. \& White, S. D. M. 1998, MNRAS, 297, L71

Steidel, C.C. 1999, PNAS, 96, 4232

Toth, G., \& Ostriker, J.P. 1992, ApJ, 389, 5

van den Bergh, S., Abraham, R. G ., Ellis, R.S., Tanvir, N. R., Santiago, B., \& Glazebrook, K. G. 1996, AJ, 112, 359

van den Bergh, S., Cohen, J., Ho gg, D. W., \& Blandford, R. 2000, AJ, 120, 2190

van den Bergh, S., Abraham, R.G ., Whyte, L.F., S. K., Ishizuki, S., Scoville, N. Z. 1999, ApJ, 525,691 